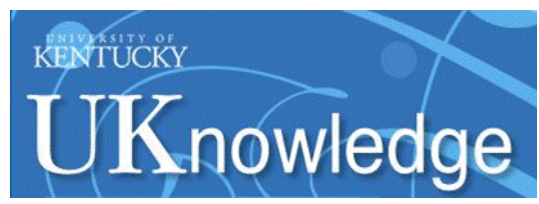

University of Kentucky

UKnowledge

8-2015

\title{
Assessment of Accelerometers for Measuring Upper-Extremity Physical Activity
}

\author{
Elizabeth Lawinger \\ University of Kentucky, elizabeth.lawinger@uky.edu \\ Timothy L. Uhl \\ University of Kentucky, tluhl2@uky.edu \\ Mark Abel \\ University of Kentucky, mgabel2@uky.edu \\ Srinath Kamineni \\ University of Kentucky, srinath.kamineni@uky.edu
}

Follow this and additional works at: https://uknowledge.uky.edu/rehabsci_facpub

Part of the Rehabilitation and Therapy Commons

Right click to open a feedback form in a new tab to let us know how this document benefits you.

\section{Repository Citation}

Lawinger, Elizabeth; Uhl, Timothy L.; Abel, Mark; and Kamineni, Srinath, "Assessment of Accelerometers for Measuring Upper-Extremity Physical Activity" (2015). Physical Therapy Faculty Publications. 48. https://uknowledge.uky.edu/rehabsci_facpub/48

This Article is brought to you for free and open access by the Physical Therapy at UKnowledge. It has been accepted for inclusion in Physical Therapy Faculty Publications by an authorized administrator of UKnowledge. For more information, please contact UKnowledge@lsv.uky.edu. 
Assessment of Accelerometers for Measuring Upper-Extremity Physical Activity

Digital Object Identifier (DOI)

http://dx.doi.org/10.1123/jsr.2013-0140

Notes/Citation Information

Published in Journal of Sport Rehabilitation, v. 24, issue 3, p. $236-243$.

(๖ 2015 Human Kinetics, Inc. as accepted for publication. http://dx.doi.org/10.1123/jsr.2013-0140

This article is available at UKnowledge: https://uknowledge.uky.edu/rehabsci_facpub/48 


\section{Note: This article will be published in a forthcoming issue of the Journal of Sport Rehabilitation. The article appears here in its accepted, peer-reviewed form, as it was provided by the submitting author. It has not been copyedited, proofed, or formatted by the publisher.}

Section: Original Research Report

Article Title: Assessment of Accelerometers for Measuring Upper Extremity Physical Activity

Authors: Elizabeth Lawinger ${ }^{1}$, Tim L. Uhl ${ }^{1}$, Mark Abel $^{2}$, and Srinath Kamineni ${ }^{3}$

Affiliations: ${ }^{1}$ Division of Athletic Training, Department of Rehabilitation Sciences, College of Health Science, University of Kentucky, Lexington, KY. ${ }^{2}$ Department of Kinesiology and Health Promotion, College of Education, University of Kentucky, Lexington, KY. ${ }^{3}$ Department of Orthopedic Surgery, College of Medicine, University of Kentucky, Lexington, KY.

Running Head: Assessment of Accelerometers

Journal: Journal of Sport Rehabilitation

Acceptance Date: March 1, 2015

(C)2015 Human Kinetics, Inc.

DOI: http://dx.doi.org/10.1123/jsr.2013-0140 
“Assessment of Accelerometers for Measuring Upper Extremity Physical Activity” by Lawinger E, Uhl TL, Abel M, Kamineni S Journal of Sport Rehabilitation

Assessment of Accelerometers for Measuring Upper Extremity Physical Activity.

Elizabeth Lawinger MS, Division of Athletic Training, Department of Rehabilitation Sciences, College of Health Science, University of Kentucky, Lexington, KY, USA.

Tim L. Uhl PhD, Division of Athletic Training, Department of Rehabilitation Sciences, College of Health Science, University of Kentucky, Lexington, KY, USA.

Mark Abel PhD, Department of Kinesiology and Health Promotion, College of Education, University of Kentucky, Lexington, KY, USA.

Srinath Kamineni MD, Associate Professor of Orthopedic Surgery, College of Medicine, University of Kentucky, Lexington, KY, USA.

There has been no presentation of this material.

There has been no financial support.

There is no conflicts of interest.

Elizabeth Lawinger, 307 Wildwood Crossings, Birmingham, AL 35211, (608)732-6385, ellawinger@gmail.com 
“Assessment of Accelerometers for Measuring Upper Extremity Physical Activity” by Lawinger E, Uhl TL, Abel M, Kamineni S Journal of Sport Rehabilitation

(C) 2015 Human Kinetics, Inc.

\section{Abstract}

Objective: The overarching goal of this study was to examine the use of tri-axial accelerometers in measuring upper extremity motions to monitor upper extremity exercise compliance. There were multiple questions investigated but the primary objective was to investigate the correlation between visually observed arm motions and tri-axial accelerometer activity counts in order to establish fundamental activity counts for the upper extremity. Study Design: Cross-sectional, Basic Research. Setting: Clinical Laboratory. Participants: Thirty healthy individuals age $=26 \pm 6$ years, body mass $=24 \pm 3 \mathrm{~kg}$, and height $=1.68 \pm 0.09 \mathrm{~m}$ volunteered. Intervention: Participants performed three series of tasks: 1) activities of daily living, 2) rehabilitation exercises 3) passive shoulder range of motion at 5 specific velocities on an isokinetic dynamometer while wearing an accelerometer on each wrist. Participants performed exercises with dominant arm to examine differences between sides. A researcher visually counted all arm motions in order to correlate counts with physical activity counts provided by the accelerometer. Main Outcome Measure: Physical activity counts derived from the accelerometer and visual observed activity counts recorded from a single investigator.

Results: There was a strong positive correlation $(r=.93, p<0.01)$ between accelerometer physical activity counts and visual activity counts for all ADL's. Accelerometers activity counts demonstrated side to side difference for all ADL's $(p<0.001)$ and 5 of the 7 rehabilitation activities $(\mathrm{p}<0.003)$. All velocities tested on the isokinetic dynamometer were shown to be significantly different from each other $(p<0.001)$. Conclusion: There is a linear relationship between arm motions counted visually and the physical activity counts generated by an accelerometer indicating that arm motions could be potentially accounted for if monitoring arm usage. The accelerometers can detect differences in relatively slow arm movement velocities which is critical if attempting to evaluate exercise compliance during early phase of shoulder rehabilitation. These results provide fundamental information that indicates that tri-axial accelerometers have the potential to objectively monitor and measure arm activities during rehabilitation and activity of daily living.

Keywords: Rehabilitation, Patient Compliance, Exercise Compliance 
“Assessment of Accelerometers for Measuring Upper Extremity Physical Activity” by Lawinger E, Uhl TL, Abel M, Kamineni S Journal of Sport Rehabilitation

(C) 2015 Human Kinetics, Inc.

Rehabilitation is a crucial component of recovery of full function following surgical interventions.[1] A full recovery is, in part, based on the patients' compliance to the required rehabilitation program. However, patients' compliance can be inadequate.[2-4] Progress and adherence to rehabilitative programs is typically determined from exercise logs which is based on subjective information provided by the patient and is potentially unreliable. $[5,6]$ A lack of compliance to a rehabilitative program can result in poor outcome, leading to patient dissatisfaction and increased cost to the health care system. $[7,8]$ Development of more objective methods to measure patient compliance would benefit the patient and health care provider to guide the rehabilitation process more accurately and may potentially result in better rehabilitation outcome. There is a need to develop instrumentation to facilitate this type of assessment. The upper extremity poses some unique challenges due to movement in multiple dimensions, the rate of motion can be very slow for some phases of rehabilitation, and the motions are asynchronous over the course of the day with various motions performed throughout a day.

A more objective measure of rehabilitation activity would allow the health care professional to observe the frequency, duration, and intensity of physical activity and exercises. An accelerometer is a small device that is attached to a patient externally and measures acceleration of limb motion and has been used to track physical activity.[9-11] Accelerometers measure rate of change during a movement and are capable of tracking three-dimensional motions which would be ideal for shoulder motions. Accelerometers have been found to provide accurate measures of physical activity in walking with an overall accuracy of $92 \%$ in patients with previous hip and knee arthroplasties.[12] Monitoring patients during rehabilitation with objective 
accelerometers may demonstrate differences in patient outcomes. Patients following hip fractures were monitored with both self-reports and accelerometers during a longitudinal study of 6 months. The objective measures from the accelerometer positively correlated $(r=.3)$ with the treating therapists rating of patients level of participation. The patients with higher accelerometer activity counts were found to have significantly higher functional outcome compared to patients with lower activity counts.[13] This indicates that greater walking activity following knee and hip surgery could be tracked and was able to discriminate the activity which had a direct bearing on functional outcome. The use of accelerometers in the upper extremity has been limited primarily to patients following a cerebral vascular accident (CVA) and found to demonstrate differences in upper extremity activity on the involved versus the uninvolved side.[14-16]

Accelerometers have the potential to more accurately measure rehabilitation compliance in upper extremity orthopedic pathologies. However, rehabilitation for orthopedic patients is quite different than rehabilitation following a CVA. In orthopedic rehabilitation, there are phases of rehabilitation that require immobilization, slow and deliberate motions to regain mobility, and then progress to more dynamic activities such as strengthening and functional activities of daily living (ADL's). It is well documented that accelerometers can collect data on typical arm movements during ADL's in a controlled setting, $[14,15,17]$ but it is not known how accelerometer output correlates to specific arm movements. Previous researchers have compared differences between arms in healthy population but have typically found no differences as individuals were allowed to perform usual activity of daily living for some period of time. $[17,18]$ The use of an accelerometer during rehabilitation would be to document patients are performing 
prescribed exercises which has not been investigated in a controlled environment previously. Furthermore, it is unclear as to the accelerometer's ability to accurately quantify slow upper extremity movements. It is important to identify the slowest velocity that the accelerometer can accurately measure. There are commonly prescribed exercises such as passive external rotation that are performed in a slow and deliberate manner that would be appropriate to capture in order to evaluate patient compliance throughout multiple rehabilitation phases. In order to use accelerometers to objectively measure upper extremity exercise compliance several fundamental questions needed to be answered first. Therefore the purpose of this study is three-fold. First, to determine the velocity threshold detectable by an accelerometer using 5 speeds on an isokinetic dynamometer which would allow us to assure that slow deliberate motions can be captured by an accelerometer. Second purpose is to determine if physical activity counts, generated by the accelerometer, can differentiate between the arm performing and not performing the activity in a controlled environment. This would simulate an injured condition of a patient performing specific exercise on one arm or not using the arm during the immobilization phase of rehabilitation. The final and most important purpose is to determine the correlation between physical activity counts generated by the accelerometer and visual activity counts recorded by the investigator to provide a context of what physical activity counts mean in relationship to number of arm movements. 
“Assessment of Accelerometers for Measuring Upper Extremity Physical Activity” by Lawinger E, Uhl TL, Abel M, Kamineni S Journal of Sport Rehabilitation

(C) 2015 Human Kinetics, Inc.

\section{Methods}

Participants

Participants for this study included 30 healthy individuals ( 21 females, 9 males) with the following demographic information (age $=26 \pm 6$ years, body mass $=24 \pm 3 \mathrm{~kg}$, and height $=1.7 \pm 0.10 \mathrm{~m}$ ). All subjects volunteered to participate by signing a university approved informed consent form. Subjects were excluded if they reported having a current upper extremity injury or previous surgical intervention to their upper extremity. Subjects were excluded if they do not have full range of motion in the shoulder, elbow, and wrist as determined by physical examination by a certified athletic trainer. Subjects hand dominance was determined using the Edinburgh Handedness Inventory.[19] Hand dominance was used to emulate one injured and one non-injured extremity.

\section{Apparatus and Measures}

Two accelerometers used in this study were ActiGraph GT3X+ (Actigraph, Pensacola, FL) activity monitor which are tri-axial accelerometers that have a mass 19 grams and physical dimensions of $4.6 \mathrm{~cm} \times 3.3 \mathrm{~cm} \times 1.5 \mathrm{~cm}$ per device. The ActiGraph GT3X+ has the ability to record accelerations in 3 dimensions and combined the three orthogonal axes by using Pythagorean's Theorem termed vector magnitude activity counts. This particular measure was used for this study as multiple plans of motion occur during rehabilitation exercises and activities of daily living of the upper extremity. The accelerometers are battery operated and were initiated on a personal computer. The investigator's watch was synchronized to the computer's internal clock prior to initiation of each data collection session to correctly record the start and end time of events during the multiple tasks described in detail below. The accelerometer sampling 
rate was set at $30 \mathrm{~Hz}$, recommended by the manufacturer, for all data collection and was attached to the wrist with wrist straps.

\section{Procedures}

The participant's age, gender, height, and body mass were recorded. The order of tests was counterbalanced using Latin square with 2 levels to minimize fatigue and order bias. The first level of counterbalancing was between 3 categories (Isokinetic, Activities of Daily Living, and Rehabilitation). The second level of counterbalancing was the specific activity within each category. The counterbalance was performed prior to enrollment for the 30 participants using an Excel (Microsoft, Redwood, WA) spreadsheet.

Accelerometers were placed on both wrists using a wrist band to prevent displacement during testing and in order to keep orientation consistent throughout testing. A single test administer instructed the subjects how to perform each activity with their specific arm, detailed below. This investigator provided all instructions and recorded all repetitions of dominant arm motions for all subjects to minimize errors. The participant was allotted 2 minutes to perform each activity while the investigator visually monitored the trial to record the trial beginning and end of each activity. This time recording was critical as it allowed the continually collected data from the accelerometers to be delineated for each specific activity. Later in data processing this allowed the specific time of an activity and the respective accelerometer physical activity counts output to be counted for each particular task. All motions were counted by the investigator with use of a video camera and recorded on a data sheet for each individual activity, regardless of the direction. For the purpose of this study these were called 
“Assessment of Accelerometers for Measuring Upper Extremity Physical Activity” by Lawinger E, Uhl TL, Abel M, Kamineni S Journal of Sport Rehabilitation

(C) 2015 Human Kinetics, Inc.

"visual activity counts". Visual activity counts were defined as the investigator's record of the number of arm motions performed by the subject. These data were used for later statistical analysis to provide context to the accelerometer's vector magnitude physical activity counts. The three tests are Speed Testing, Activity of Daily Living Testing, and Rehabilitation Testing and are detailed below.

\section{Speed Testing}

Isokinetic testing was performed with the participant in supine position with the shoulder flexed to $90^{\circ}$. The subject was instructed to grasp onto the handle of an isokinetic dynamometer (Cybex Norm, Stoughton, MA). The dynamometer moved the arm passively at 5 different velocities $\left(0.5,15,30,45,60^{\circ} \cdot \mathrm{s}^{-1}\right)$, with each angular velocity serving as a trial. The subject performed 2 minutes of passive shoulder flexion at each angular velocity through shoulder flexion range of motion of 0 to $90^{\circ}$. The investigator video recorded and then visually counted and recorded the number of dominant arm motions during the trial on the data sheet. The subject was instructed to allow their non-dominant arm to rest by their side during testing. There was a recovery period of one minute between trials to set the next velocity on the isokinetic dynamometer. Testing was repeated until all 5 velocities were recorded.

\section{Activities of Daily Living Testing}

The test administrator explained the four activities of daily living (ADL) to the subjects prior to each task. The ADL's were selected based on their use in previous publications. [20, 21] Nine dinner bowls were moved from the countertop to the second shelf of an overhead cabinet with the dominant arm only. Washing a countertop by spraying a .9 by $.65 \mathrm{~m}$ area with a standard surface cleaning product with the non- 
dominant arm and wiping clean until dry with the dominant arm. Subjects vacuumed a .9 by 1.5 m carpet area with a Panasonic upright electric vacuum (MC-V5210, Secaucus, NJ) with the dominant arm. Washing a mirror by spraying a .53 by $1.65 \mathrm{~m}$ area mirror with the non-dominant arm and wiping clean until dry with the dominant arm. The specific manner in which to carry out the tasks was up to the individual in order to simulate real life. The only control was the time limit and the arm used to perform the task.

\section{Rehabilitation Testing}

The rehabilitation exercises were selected based on the standard rehabilitation program for a rotator cuff repair from prescribed by the investigators and from the literature. [22-24] The test administrator explained and demonstrated each exercise prior to having the subject perform the seven rehabilitation exercises with the dominant arm. The exercises performed were: passive pendulum exercises, standing passive external rotation, passive internal rotation towel stretches, passive forward bows, active assistive table slides, resistive internal rotation, and resistive rows with elastic band.[2224] All subjects were instructed to perform 20 repetitions of each exercise. The number of actual repetitions performed by the subject's dominant arm was counted by a single investigator from the video record.

\section{Data Reduction}

All visual recording of dominant arm motion was transferred from data sheet to excel file. Activities of daily living were watched a minimum of three times using a video camera to correctly record visual activity counts which allowed participants the most freedom in arm movements. The vector magnitude physical of activity counts from each 
accelerometer were calculated with the ActiLife software (Actigraph, Pensacola, FL). The ActiLife vector magnitude physical activity counts were exported to Exce spreadsheet using 1 second epoch lengths for every second during the entire data collection. This value could range from 0 to several hundred counts depending on the magnitude of the acceleration. Each row of data represented one second of physical activity counts. The start and end time of each activity recorded on the data sheet were identified by a single investigator. A blinded investigator summed the total vector magnitude physical activity counts for both arms individually for each of the three tests. This data was used for the statistical analysis along with the visual activity counts recorded.

Statistical Analysis

To determine if the accelerometer physical activity counts can differentiate between speeds during isokinetic testing, the dominant arm accelerometer physical activity counts were analyzed with a repeated measure ANOVA with one within variable of speed with 5 levels. The non-dominant data was not used in this analysis as the question was between velocities not differences between arms. To determine if the accelerometer physical activity counts can differentiate between the dominant and nondominant arm movements during activities of daily living and rehabilitation exercises, two separate repeated measure ANOVAs were used. The repeated measures ANOVA for activities of daily living had two within variables; activity (4 levels) by arm (2 levels). The repeated measures ANOVA for rehabilitation exercises had two within variables; exercises ( 7 levels) by arm (2 levels). For all measures alpha was set a priori at $p \leq .05$. 
For significant difference, Bonferroni post-hoc analyses were performed to determine where specific differences occurred with a p value corrected for multiple comparisons.

To investigate the context of what physical activity counts mean in relationship to number of arm movements, a bivariate correlation was performed between the visual activity counts and the vector magnitude for activities of daily living to determine the relationship between the accelerometer physical activity counts and visual activity counts.

Results

Mauchly's sphericity test was significant for all repeated measure ANOVAs. The Greenhouse-Geisser correction was used as the epsilon was $<0.75$ to correct for the lack of homogeneity of variance for all the below results.[25] The first purpose of this study determined which speeds could be detected by the accelerometer. There was a significant difference between all velocities $(p<.001)$. Post-hoc analysis with a Bonferroni correction revealed that at each velocity, the physical activity counts were different for the dominant arm. (Figure 1)

The second purpose of this study was to determine if the physical activity counts generated by the accelerometers could identify differences between the arm performing and not performing the specific activity in a controlled environment. There was a significant interaction for activity by arm for ADLs $(p<.001)$ and a significant interaction for exercise by arm for rehabilitation exercises $(p<.001)$. A Bonferroni post-hoc analysis demonstrated the dominant arm was always more active for all ADLs.(Table 1 ) A Bonferroni post-hoc analysis demonstrated that the dominant arm was more active in 5 of the 7 rehabilitation exercises. (Table 2) The external rotation activity revealed no 
difference between the two arms $(p=.18)$. The towel internal rotation stretch activity

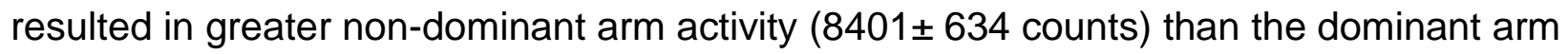
(7116 \pm 463 counts $)(p=.016)$.

The third purpose was to determine the correlation between physical activity counts generated by the accelerometer and visual activity counts recorded by the investigator to provide a context of what physical activity counts mean in relationship to arm movements. The bivariate correlation of the dominant arm accelerometer with 3 of the 4 ADLs (vacuuming, washing mirror and countertop activities combined) resulted in a significant correlation of $(r=.93, p<.001)$. (Figure 2$)$ The shelving bowls activity, rehabilitation exercises, and isokinetic testing data were not considered as each of these activities had been given purposeful constant counts or time which would invalidate a correlation calculation.

\section{Discussion}

This study provides fundamental information regarding the use of accelerometers for objectively capturing upper extremity movements. The results of this study suggest that accelerometers can differentiate activity between relatively slow motions. This confirms the previous finding that accelerometers can differentiate between arms in a controlled setting $[14,15]$ and provides new information regarding the context of accelerometers physical activity counts in the upper extremity.

Acceleration is the rate of change of velocity over the change in time. Therefore, an isokinetic measure which moves nearly at a constant rate would be a good instrument to determine the speeds at which the ActiGraph GT3X+could differentiate quantified motions. The current study results indicate that arm movements can be 
detected by the ActiGraph GT3X+. This is important, as many shoulder rehabilitation exercises are performed in a relatively slow and deliberate manner. All movements were shown to be significantly different than each other: therefore the ActiGraph GT3X+ is able to capture differences in velocities during arm movement. A secondary analysis was done comparing the dominant arm and the non-dominant arm motion at each velocity. There was significantly more activity in the dominant arm compared to the nondominant arm at speeds equal to and above $30^{\circ} \cdot \mathrm{s}^{-1}(\mathrm{p} .<.001)$. At $15^{\circ} \cdot \mathrm{s}^{-1}$ there was no difference between the arms $(p=.54)$. The movements at $.5^{\circ} \cdot \mathrm{s}^{-1}$ were found to be so slow that they were actually less than the non-dominant arm $(p=.011)$ which moved minimally during the testing. Subjects were instructed to relax their arm but their nondominant was not strapped down to prevent any motion, which may account for the increase of non-dominant arm motion. The non-dominant arm motion physical activity counts was not different through all testing speeds $(p>.28)$.

Accelerometers measure rate of change not the amount of motion occurring. As several rehabilitation exercises following shoulder surgery are performed in a slow and deliberate manner, it is important to know if the accelerometers can detect relatively slow movements. One of the most basics and commonly prescribed post-operative shoulder rehabilitation exercises is the pendulum exercise. The accelerometer was able to clearly differentiate between arms performing this exercise in a healthy population. This cannot be directly extrapolated to an injured population as their rate of motion may be slower. However, the relatively slow speeds able to be detected produce promising initial results in the use of accelerometers to track exercise compliance. The typical velocity of activities of daily living certainly varies by task and individual but in a study by 
“Assessment of Accelerometers for Measuring Upper Extremity Physical Activity” by Lawinger E, Uhl TL, Abel M, Kamineni S Journal of Sport Rehabilitation

(C) 2015 Human Kinetics, Inc.

Lacquaniti it was found that reaching velocities ranged from $80^{\circ}$ to $200^{\circ} \cdot \mathrm{s}^{-1}[26]$. The current study demonstrated that much slower velocities can be detected by the ActiGraph GT3X+ accelerometers.

Accelerometers were shown to be able to detect differences between the dominant and non-dominant arm when the dominant arm is the prime mover during the activity. These results were found with all activities of daily living and five out of the seven rehabilitation exercises. The study was set up to facilitate this result due to instructions given to the subjects. The intention was to simulate a condition with one injured and one non-injured extremity to determine if utilization of arms could be detected. The ability to differentiate between arms agrees with previous research of hemiparetic population, when the affected arm was shown to be used less than the nonaffected limb.[14] Hemiparetic patients have been shown to use their affected and unaffected arm less than a healthy population. In addition, their affected arm is used only 3.3 hours per day compared to their unaffected arm at 6.0 hours per day.[14] Supporting that arm activity can be discriminated, however in unrestricted activity of a healthy population there was no difference between the physical activity counts between arms.[14, 17] Future research is needed to determine if difference between injured and non-injured arms can detect similar difference as found in hemiparetic patients.

Two rehabilitation exercises did not have more activity in the dominant arm. This can be explained by the way the exercises were performed. The towel stretch requires both arms to perform the exercise simultaneously, however the dominant arm was behind the subject's back which may have impaired motion of the accelerometer. The 
direct contact between the subject's body and the dominant accelerometer may have reduced some of the motion occurring resulting in lower physical activity counts. The external rotation stretch requires the subject to hold their dominant arm on a door frame and rotate their body around the arm. This would require movement of the nondominant arm as it moves with the body which likely explains that there was no differences in the physical activity counts between arms during this exercise. The rate of arm motion was not controlled but this exercise was among the slowest movement performed and produced one of the lowest physical activity counts.(Table 2) The lower velocity of motion may also account for the lack of difference between the arms.

The accelerometers could discriminant between 9/11 (81\%) of the activities performed in this study but it cannot discriminate between all activities. The prescript laboratory environment is far from post-operative shoulder patients functioning independently at home with precautions and specified rehabilitation exercises. The discriminating nature of these results suggests accelerometers may provide a useful tool to objectively measure exercise activity in the future. These results are encouraging and support further investigation, in patients with a shoulder injury, to determine if they are resting or not resting their injured arm as prescribed by their treating physician.

The physical activity counts of a uniaxial accelerometer have been correlated to the number of steps a person takes over the course of a day. There are even recommended step counts for a healthy lifestyle.[27, 28] In the upper extremity, the use of accelerometers is far behind that of the lower extremity. There is limited research but has primarily focused on difference between arms and energy expenditures. One intention of this study was to provide contextual information as to what does a physical 
activity count mean for the upper extremity. Therefore, a correlation between physical activity counts to accelerometer counts was undertaken. The results of the current study show a strong positive nearly linear correlation of $r=.93(p<.01), R^{2}=.87$. This indicates that there is a relatively linear relationship between upper extremity activities of daily living visual counts and accelerometer physical activity counts. This is an initial step to understand categories of arm movements similar to how many steps per day should be taken for an active lifestyle. The linearity of this relationship allows for context to be given to the vector magnitude physical activity counts of GT3X+accelerometer. The context derived in this controlled laboratory study resulted that for every 100 vector magnitude physical activity counts equals 5 arm motions. This is just a first step in a series of studies that needs to occur before we can categorize arm motions similar to lower extremity steps per day into categories of activities based on counts. [27, 29, 30] This study has limitations that we fully acknowledge. The test sessions were conducted in a laboratory setting with healthy population. These results may not be extrapolated to be indicative of a person's normal day-to-day activities or how an injured individual may use their upper extremity. Activities measured in this study were performed in healthy population so the rate of motion cannot be extrapolated to injured population as they may perform activities at a very different rate which would directly affect the physical activity counts. The context of physical activity counts cannot be extrapolated to an injured population until further research is performed. Also, when assessing the activities of daily living and rehabilitation activities we used physical activity counts. Physical activity counts have been shown to have a relationship with the 
“Assessment of Accelerometers for Measuring Upper Extremity Physical Activity” by Lawinger E, Uhl TL, Abel M, Kamineni S Journal of Sport Rehabilitation

(C) 2015 Human Kinetics, Inc.

lower extremity activity but this is the first study to show this relationship in the upper extremity.

Conclusions

This study's findings suggest that ActiGraph GT3X+ accelerometers have a good potential to be a valid tool for measuring exercise compliance based on the results of this fundamental study that was performed in a controlled laboratory setting. This study addressed three primary purposes that provide fundamental information for use of accelerometers in measuring upper extremity activity to evaluate exercise compliance. ActiGraph GT3X+ accelerometers can detect differences in varying arm movement velocities on an isokinetic dynamometer. The accelerometers can detect differences in relatively slow arm movement velocities which is critical if attempting to evaluate exercise compliance during the early phase of shoulder rehabilitation. The accelerometers vector magnitude physical activity counts can be used to discriminant between arms for most specific arm activities in a controlled laboratory environment. This is important in order to confirm patients were compliant if given instruction to perform varying amounts unilateral and bilaterally exercises we would expect to see a difference between the two accelerometer counts indicating compliance to the exercises prescribed. There is a nearly linear relationship between vector magnitude physical activity counts generated by the accelerometer and visually observed arm motions. These results suggest that a link between arm motions could be determine by having patients wear an accelerometer during rehabilitation to objectively measure arm usage over the course of days or weeks. These results support the need for further research in supervised and unsupervised environments, on patient with upper extremity 
“Assessment of Accelerometers for Measuring Upper Extremity Physical Activity” by Lawinger E, Uhl TL, Abel M, Kamineni S Journal of Sport Rehabilitation

(C) 2015 Human Kinetics, Inc.

pathologies, to further determine if accelerometers can provide objective measure of arm use during activities of daily living and rehabilitation, in order to more objectively track exercise compliance. 
“Assessment of Accelerometers for Measuring Upper Extremity Physical Activity” by Lawinger E, Uhl TL, Abel M, Kamineni S Journal of Sport Rehabilitation

(C) 2015 Human Kinetics, Inc.

\section{References}

1. Brems, J.J., Rehabilitation following total shoulder arthroplasty. Clinical Orthopaedics And Related Research, 1994(307): p. 70-85.

2. Petersen, S.A. and R.J. Hawkins, Revision of failed total shoulder arthroplasty. The Orthopedic Clinics Of North America, 1998. 29(3): p. 519-533.

3. Roddey, T.S., et al., A randomized controlled trial comparing 2 instructional approaches to home exercise instruction following arthroscopic full-thickness rotator cuff repair surgery. The Journal Of Orthopaedic And Sports Physical Therapy, 2002. 32(11): p. 548-559.

4. Popowitz, R.L., et al., Rotator cuff repair in spinal cord injury patients. J Shoulder Elbow Surg, 2003. 12(4): p. 327-32.

5. Zahiri, C.A., et al., Assessing activity in joint replacement patients. The Journal Of Arthroplasty, 1998. 13(8): p. 890-895.

6. Dawson, J., et al., Comparison of clinical and patient-based measures to assess medium-term outcomes following shoulder surgery for disorders of the rotator cuff. Arthritis And Rheumatism, 2002. 47(5): p. 513-519.

7. Razmjou, H., et al., Factors contributing to failure of rotator cuff surgery in persons with workrelated injuries. Physiotherapy Canada. Physiothérapie Canada, 2008. 60(2): p. 125-133.

8. Djurasovic, M., et al., Revision rotator cuff repair: factors influencing results. J Bone Joint Surg Am, 2001. 83-A(12): p. 1849-55.

9. Vanhelst, J., et al., Validation of the Vivago Wrist-Worn accelerometer in the assessment of physical activity. BMC Public Health, 2012. 12: p. 690-690.

10. Ekblom, O., et al., Validity and comparability of a wrist-worn accelerometer in children. Journal Of Physical Activity \& Health, 2012. 9(3): p. 389-393.

11. Shaoyan, Z., et al., Physical Activity Classification Using the GENEA Wrist-Worn Accelerometer. Medicine \& Science in Sports \& Exercise, 2012. 44(4): p. 742-748.

12. Schmalzried, T.P., et al., Quantitative assessment of walking activity after total hip or knee replacement. The Journal Of Bone And Joint Surgery. American Volume, 1998. 80(1): p. 54-59.

13. Talkowski, J.B., et al., Patient participation and physical activity during rehabilitation and future functional outcomes in patients after hip fracture. Arch Phys Med Rehabil, 2009. 90(4): p. 61822.

14. Lang, C.E., et al., Upper extremity use in people with hemiparesis in the first few weeks after stroke. Journal of Neurologic Physical Therapy, 2007. 31(2): p. 56-63.

15. van der Pas, S.C., et al., Assessment of arm activity using triaxial accelerometry in patients with a stroke. Arch Phys Med Rehabil, 2011. 92(9): p. 1437-42. 
“Assessment of Accelerometers for Measuring Upper Extremity Physical Activity” by Lawinger E, Uhl TL, Abel M, Kamineni S Journal of Sport Rehabilitation

(C) 2015 Human Kinetics, Inc.

16. Uswatte, G., et al., Objective measurement of functional upper-extremity movement using accelerometer recordings transformed with a threshold filter. Stroke, 2000. 31(3): p. 662-7.

17. Acuna, M., T. Amasay, and A.R. Karduna, The reliability of side to side measurements of upper extremity activity levels in healthy subjects. BMC Musculoskelet Disord, 2010. 11: p. 168.

18. Acuna, M. and A.R. Karduna, Wrist activity monitor counts are correlated with dynamic but not static assessments of arm elevation exposure made with a triaxial accelerometer. Ergonomics, 2012. 55(8): p. 963-970.

19. Ransil, B.J. and S.C. Schachter, Test-retest reliability of the Edinburgh Handedness Inventory and Global Handedness preference measurements, and their correlation. Perceptual And Motor Skills, 1994. 79(3 Pt 1): p. 1355-1372.

20. Swartz, A.M., et al., Estimation of energy expenditure using CSA accelerometers at hip and wrist sites. Med Sci Sports Exerc, 2000. 32(9 Suppl): p. S450-6.

21. Eisenmann, J.C., et al., Validity of uniaxial accelerometry during activities of daily living in children. Eur J Appl Physiol, 2004. 91(2-3): p. 259-63.

22. Conti, M., et al., Post-operative rehabilitation after surgical repair of the rotator cuff. La Chirurgia Degli Organi Di Movimento, 2009. 93 Suppl 1: p. S55-S63.

23. Duzgun, I., G. Baltaci, and O.A. Atay, Comparison of slow and accelerated rehabilitation protocol after arthroscopic rotator cuff repair: pain and functional activity. Acta Orthop Traumatol Turc, 2011. 45(1): p. 23-33.

24. Fleming, J.A., A.L. Seitz, and D.D. Ebaugh, Exercise protocol for the treatment of rotator cuff impingement syndrome. J Athl Train, 2010. 45(5): p. 483-5.

25. Field, D.A., Repeated Measures ANOVA. Research Methods in Psychology, 2008: p. 1-23.

26. Lacquaniti F, S.J., Coordination of Arm and Wrist Motion During A Reaching Task. The Journal of Neuroscience, 1982. 2(4): p. 9.

27. Catrine Tudor-Locke, 2 Cora L Craig,2,3 Wendy J Brown,4 Stacy A Clemes,5 Katrien De Cocker,6 Billie Giles-Corti,7 Yoshiro Hatano,8 Shigeru Inoue,9 Sandra M Matsudo,10 Nanette Mutrie,11 Jean-Michel Oppert,12 David A Rowe,11 Michael D Schmidt,13,14 Grant M Schofield,15 John C Spence,16 Pedro J Teixeira,17 Mark A Tully,18 and Steven N Blair, How Many Steps/day are Enough? For Adults. International Journal of Behavioral Nutrition and Physical Activity, 2011. 8(79): p. 17.

28. Bohannon, R.W., Number of pedometer-assessed steps taken per day by adults: a descriptive meta-analysis. Physical Therapy, 2007. 87(12): p. 1642-1650.

29. Tudor-Locke, C., W.D. Johnson, and P.T. Katzmarzyk, Accelerometer-Determined Steps per Day in US Adults. Medicine \& Science in Sports \& Exercise, 2009. 41(7): p. 1384-1391.

30. Tudor-Locke, C. and D.R. Bassett Jr, How Many Steps/Day Are Enough?: Preliminary Pedometer Indices for Public Health. Sports Medicine, 2004. 34(1): p. 1-8. 


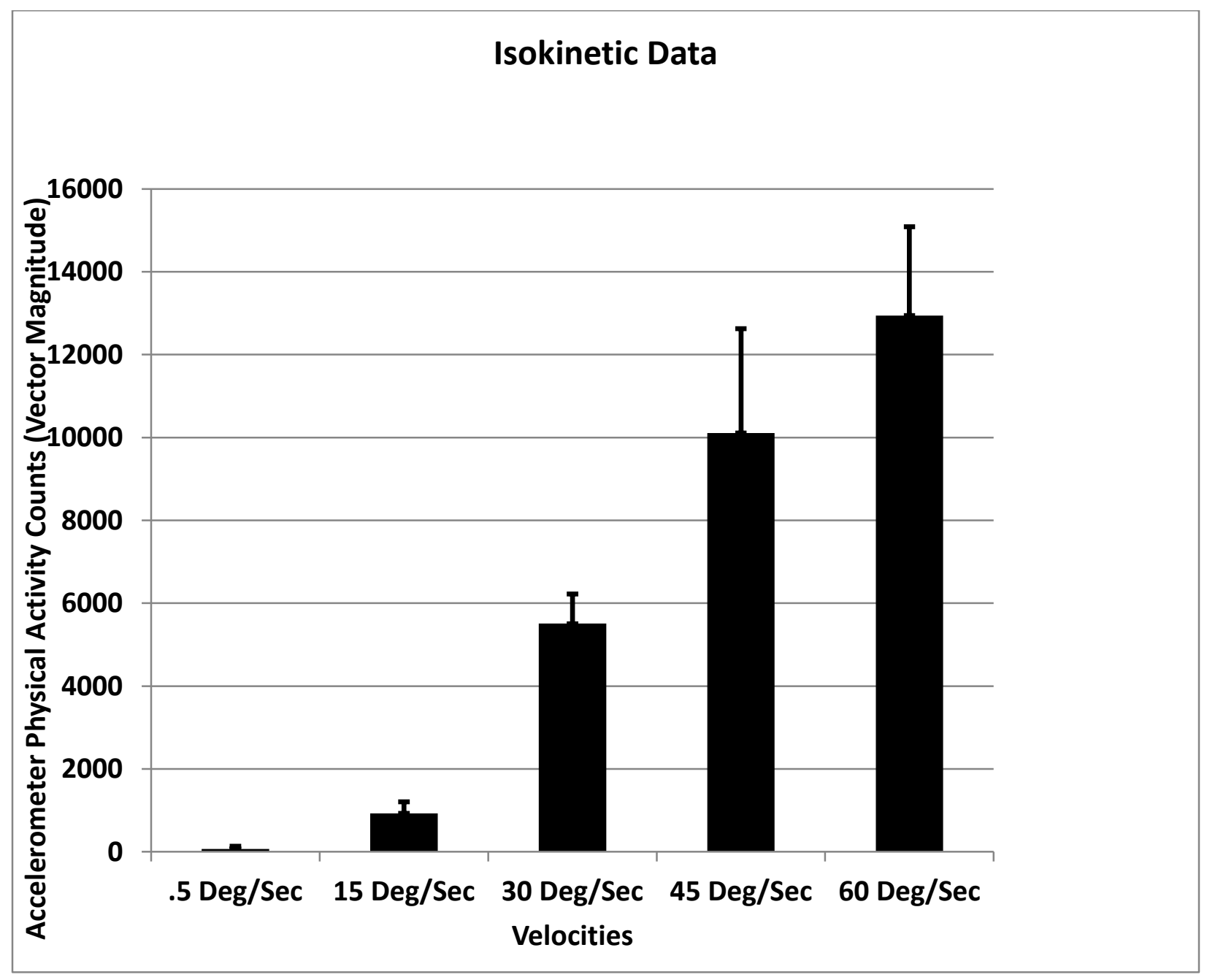

Figure 1. The figure illustrates the difference between the dominant arm's physical activity counts at the 5 different passive speeds on the isokinetic dynamometer. Bonferroni post-hoc analysis revealed a significant difference at each increasing speed for the dominant arm $(p<.001)$. 


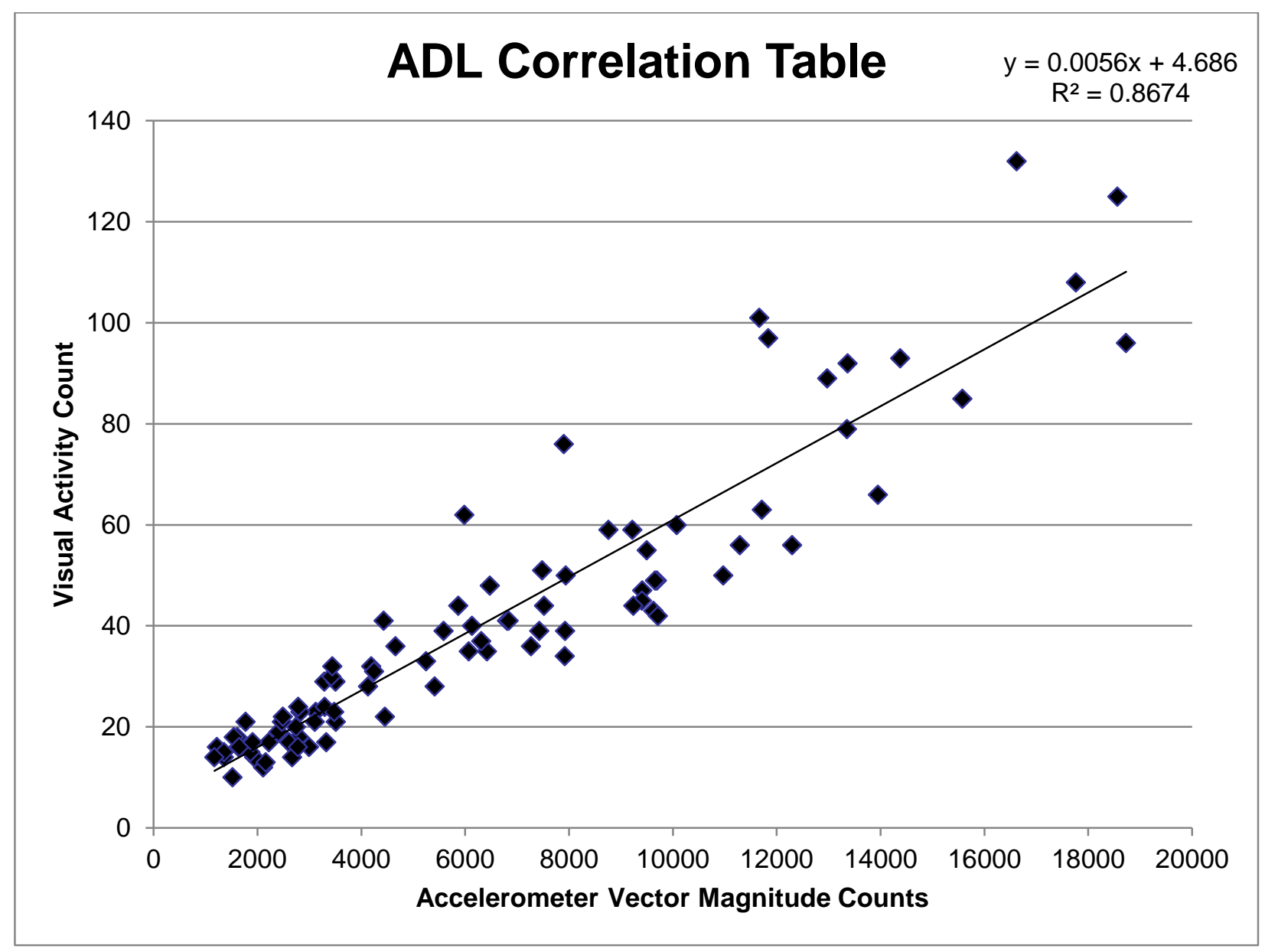

Figure 2. Scatterplot of all ADL with vector magnitudes physical activity counts on the $X$ axis to predict the visual activity counts. The relatively linear relationship produces an $\mathrm{R}^{2}=.867$ and indicates that for every 4000 vector magnitude physical activity counts equal 27 arm motions. 
“Assessment of Accelerometers for Measuring Upper Extremity Physical Activity” by Lawinger E, Uhl TL, Abel M, Kamineni S Journal of Sport Rehabilitation

(C) 2015 Human Kinetics, Inc.

Table 1. Activities of Daily Living results demonstrate more activity in the dominant arm than the non-dominant arm in the 30 subjects.

\begin{tabular}{cccccc}
\hline & Dominant & \multicolumn{3}{c}{ Non- } & Significance \\
Activity & MN & SD & MN & SD & P \\
Plates & 5,423 & 509 & 141 & 246 & $<.001$ \\
Vacuum & 2,618 & 1,378 & 1,696 & 912 & $<.001$ \\
Mirror & 11,550 & 3,478 & 1,533 & 583 & $<.001$ \\
Counter top & 5,174 & 2,015 & 806 & 486 & $<.001$ \\
\hline
\end{tabular}


“Assessment of Accelerometers for Measuring Upper Extremity Physical Activity” by Lawinger E, Uhl TL, Abel M, Kamineni S Journal of Sport Rehabilitation

(C) 2015 Human Kinetics, Inc.

Table 2. Results of rehabilitation exercises demonstrating more activity in the dominant arm than the non-dominant arm for all activities except external rotation stretch and towel stretch.

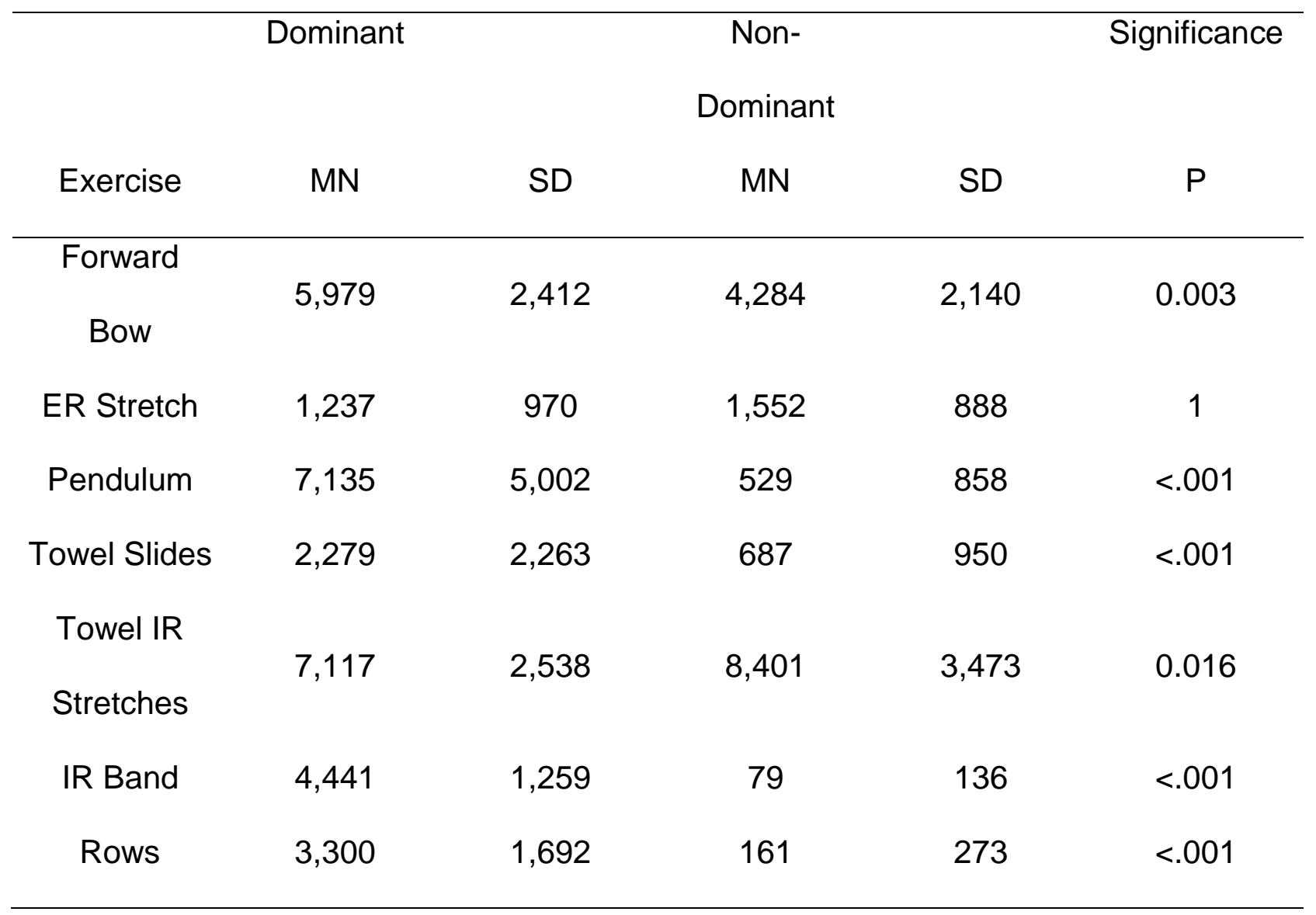

